\title{
Generalized Vandermonde determinants
}

\author{
by \\ Hans Peter Schlickewei (Marburg) and Carlo Viola (Pisa)
}

1. Introduction. Let $k \geq 2$ be an integer, and let $\alpha_{1}, \ldots, \alpha_{k}$ be non-zero elements in a field of characteristic zero. Define the function $F\left(X_{1}, \ldots, X_{k}\right)$ in integral variables $X_{1}, \ldots, X_{k}$ by

$$
F\left(X_{1}, \ldots, X_{k}\right)=\left|\begin{array}{ccc}
\alpha_{1}^{X_{1}} & \ldots & \alpha_{k}^{X_{1}} \\
\ldots \ldots & \ldots & \ldots \\
\alpha_{1}^{X_{k}} & \ldots & \alpha_{k}^{X_{k}}
\end{array}\right| .
$$

$F(0,1, \ldots, k-1)$ is the usual Vandermonde determinant and it is well known that

$$
F(0,1, \ldots, k-1)=\prod_{1 \leq i<j \leq k}\left(\alpha_{j}-\alpha_{i}\right) .
$$

In particular, $F(0,1, \ldots, k-1)=0$ if and only if there exists a pair $i, j$ with $1 \leq i<j \leq k$ such that $\alpha_{i}=\alpha_{j}$.

We say that $F\left(X_{1}, \ldots, X_{k}\right)$ is non-degenerate if for any pair $i, j$ with $1 \leq i<j \leq k$ the ratio $\alpha_{i} / \alpha_{j}$ is not a root of unity.

It is the purpose of the present paper to study the equation

$$
F\left(x_{1}, \ldots, x_{k}\right)=0
$$

with $x_{1}, \ldots, x_{k} \in \mathbb{Z}$ and $F$ non-degenerate.

Our definition (1.1) shows that $F$ satisfies the functional equation

$$
F\left(X_{1}, \ldots, X_{k}\right)=\left(\alpha_{1} \ldots \alpha_{k}\right)^{X_{1}} F\left(0, X_{2}-X_{1}, \ldots, X_{k}-X_{1}\right) .
$$

Therefore, in studying (1.2) it suffices to study

$$
F\left(0, y_{2}, \ldots, y_{k}\right)=0
$$

with $y_{2}, \ldots, y_{k} \in \mathbb{Z}$.

We say that a solution $\left(y_{2}, \ldots, y_{k}\right) \in \mathbb{Z}^{k-1}$ of $(1.3)$ is in general position

2000 Mathematics Subject Classification: 11D61, 11 B37. 
if in the corresponding determinant

$$
\left|\begin{array}{ccc}
1 & \ldots & 1 \\
\alpha_{1}^{y_{2}} & \ldots & \alpha_{k}^{y_{2}} \\
\ldots \ldots \ldots & \ldots \\
\alpha_{1}^{y_{k}} & \ldots & \alpha_{k}^{y_{k}}
\end{array}\right|
$$

no proper subdeterminant vanishes. We prove

TheOREM 1.1. Let $\alpha_{1}, \ldots, \alpha_{k}$ be non-zero elements in a field of characteristic zero. Suppose that the function $F\left(X_{1}, \ldots, X_{k}\right)$ defined by (1.1) is non-degenerate. Then equation (1.3) does not have more than

$$
\exp \left((6 k !)^{3 k !}\right)
$$

solutions $\left(y_{2}, \ldots, y_{k}\right) \in \mathbb{Z}^{k-1}$ in general position.

The significant feature in our bound (1.5) is that it depends only on the dimension $k$ but is completely independent of $\alpha_{1}, \ldots, \alpha_{k}$.

Our proof uses a recent result of Evertse, Schlickewei and Schmidt [4] on linear equations over finitely generated multiplicative groups, and therefore implicitly it is an application of the subspace theorem in diophantine approximation.

It is plausible that, under the assumptions of Theorem 1.1, equation (1.3) has only finitely many solutions $\left(y_{2}, \ldots, y_{k}\right) \in \mathbb{Z}^{k-1}$ satisfying some condition weaker than the above. We state the following

Conjecture. Let $\alpha_{1}, \ldots, \alpha_{k}$ and $F\left(X_{1}, \ldots, X_{k}\right)$ be as in Theorem 1.1. Then equation (1.3) has at most finitely many solutions $\left(y_{2}, \ldots, y_{k}\right) \in \mathbb{Z}^{k-1}$ such that in the corresponding determinant $(1.4)$ all $(k-1) \times k$ and all $k \times(k-1)$ submatrices have rank $k-1$.

We postpone the proof of Theorem 1.1 to Sections 4-6.

In the next two sections we apply Theorem 1.1 to linear recurrence sequences.

2. Applications to recurrence sequences. For $k \geq 2$ we consider linear recurrence sequences $\left(u_{n}\right)_{n \in \mathbb{Z}}$ of order $k$, i.e., sequences satisfying a relation

$$
u_{n+k}=\nu_{k-1} u_{n+k-1}+\ldots+\nu_{0} u_{n} .
$$

For simplicity, we assume here that $\nu_{0}, \ldots, \nu_{k-1}$ are given elements in an algebraically closed field $\mathbb{K}$ of characteristic zero, and that $\nu_{0} \neq 0$. To avoid trivialities, we also assume that the sequence has initial values $u_{0}, \ldots, u_{k-1} \in$ $\mathbb{K}$ not all equal to zero. Let

$$
G(z)=z^{k}-\nu_{k-1} z^{k-1}-\ldots-\nu_{0}=\prod_{i=1}^{k}\left(z-\alpha_{i}\right)
$$


be the companion polynomial of the recurrence relation (2.1). The roots $\alpha_{i}$ of $G(z)$ are called the characteristic roots of the recurrence sequence $\left(u_{n}\right)$. We restrict ourselves to sequences $\left(u_{n}\right)$ with simple characteristic roots $\alpha_{i}$. So in (2.2) we assume that

$$
\alpha_{i} \neq \alpha_{j} \quad \text { for all } i, j \text { with } 1 \leq i<j \leq k .
$$

Notice that our hypothesis $\nu_{0} \neq 0$ implies

$$
\alpha_{1} \ldots \alpha_{k} \neq 0 .
$$

It is well known that under these assumptions our sequence has a representation

$$
u_{n}=a_{1} \alpha_{1}^{n}+\ldots+a_{k} \alpha_{k}^{n} \quad(n \in \mathbb{Z})
$$

with certain coefficients $a_{i} \in \mathbb{K}$, not all equal to zero, depending on the initial values $u_{0}, \ldots, u_{k-1}$. Vice versa, given $\alpha_{1}, \ldots, \alpha_{k} \in \mathbb{K}$ as in (2.3), (2.4), define $\nu_{0}, \ldots, \nu_{k-1}$ by relation (2.2). Then for any choice of $a_{1}, \ldots, a_{k} \in \mathbb{K}$ not all zero, the sequence $\left(u_{n}\right)_{n \in \mathbb{Z}}$ obtained via $(2.5)$ is a non-trivial solution of the recurrence relation $(2.1)$.

In the sequel we will suppose that our sequence $\left(u_{n}\right)$ has minimal order $k$, i.e., that it does not satisfy a non-trivial relation (2.1) of order $<k$. This is equivalent to the assumption that in (2.5),

$$
a_{1} \ldots a_{k} \neq 0 \text {. }
$$

The zero-multiplicity $M(0)$ of our sequence is defined as the number of solutions $n \in \mathbb{Z}$ of the equation

$$
u_{n}=0 .
$$

The theorem of Skolem-Mahler-Lech implies the following: Suppose $\left(u_{n}\right)$ is a linear recurrence sequence of order $k$ with infinite zero-multiplicity $M(0)$. Then there exists a pair $i, j$ with $1 \leq i<j \leq k$ such that $\alpha_{i} / \alpha_{j}$ is a root of unity. Accordingly, the sequence $\left(u_{n}\right)$ is called non-degenerate if for each pair $i, j(1 \leq i<j \leq k)$,

$$
\alpha_{i} / \alpha_{j} \text { is not a root of unity. }
$$

In [4], Evertse, Schlickewei and Schmidt have shown that, for any nondegenerate sequence $\left(u_{n}\right)$ of order $k$ with simple characteristic roots as above, we have

$$
M(0)<\exp \left(2(6 k)^{3 k}\right) .
$$

For further references on this problem, the reader is referred to [4].

Our result in Theorem 1.1 implies that in most cases much more than (2.9) is true. To discuss what may be expected, suppose first that in (2.5) the coefficients $a_{1}, \ldots, a_{k}$ are real numbers and that $\alpha_{1}, \ldots, \alpha_{k}$ are positive 
numbers. Under these assumptions, it is easy to show that the equation

$$
a_{1} \alpha_{1}^{x}+\ldots+a_{k} \alpha_{k}^{x}=0
$$

even has at most $k-1$ solutions $x \in \mathbb{R}$. Thus in particular, under the above restrictions, for equation (2.7) we get

$$
M(0) \leq k-1 .
$$

We briefly give the argument for this assertion, using induction on $k$. For $k=2$, writing $\beta=\alpha_{1} / \alpha_{2}, a=-a_{2} / a_{1}$, equation (2.10) becomes $\beta^{x}=a$ with $\beta>0, \beta \neq 1, a \in \mathbb{R}$. So we get at most one solution $x \in \mathbb{R}$.

Now suppose $k>2$ and our assertion on equation (2.10) to be true for $k-1$. We write $(2.10)$ as

$$
a_{1} \beta_{1}^{x}+\ldots+a_{k-1} \beta_{k-1}^{x}+a_{k}=0,
$$

with $\beta_{i}=\alpha_{i} / \alpha_{k}$. The derivative of the left-hand side of (2.12) is

$$
\left(a_{1} \log \beta_{1}\right) \beta_{1}^{x}+\ldots+\left(a_{k-1} \log \beta_{k-1}\right) \beta_{k-1}^{x} .
$$

By the inductive assumption, it has at most $k-2$ real zeros. But then Rolle's theorem implies that (2.12) has at most $k-1$ real solutions.

As a consequence of Theorem 1.1, we prove that in general, apart from a few exceptional cases, even when the $a_{i}$ and $\alpha_{i}$ are not contained in $\mathbb{R}$ the bound given in (2.11) essentially remains true.

Given two linear recurrence sequences $\left(u_{n}\right)$ and $\left(v_{n}\right)$ of order $k$, we say that $\left(u_{n}\right)$ and $\left(v_{n}\right)$ are equivalent if there exist $a \in \mathbb{K}, a \neq 0$, and an integer $h$ such that

$$
v_{n}=a u_{n+h} \quad \text { for all } n \in \mathbb{Z} .
$$

Writing $M_{u}(0)$ and $M_{v}(0)$ for the zero-multiplicities of $\left(u_{n}\right)$ and $\left(v_{n}\right)$ respectively, it is clear that for equivalent sequences we have $M_{u}(0)=M_{v}(0)$. In fact, if $\left(u_{n}\right)$ and $\left(v_{n}\right)$ are equivalent there is a bijection between the set of zeros of $\left(u_{n}\right)$ and the set of zeros of $\left(v_{n}\right)$. Therefore, it makes sense to speak of the zero-multiplicity of an equivalence class.

Also, equivalent sequences clearly satisfy the same recurrence relation (2.1) and have the same characteristic roots $\alpha_{1}, \ldots, \alpha_{k}$.

Fix $\alpha_{1}, \ldots, \alpha_{k} \in \mathbb{K}$ with $(2.4)$ and (2.8), and let $\left(u_{n}\right)$ be given by (2.5) with coefficients $a_{1}, \ldots, a_{k} \in \mathbb{K}$ satisfying (2.6). If $n_{1}, \ldots, n_{k}$ are solutions of (2.7), in view of (2.5) we have

$$
\left|\begin{array}{ccc}
\alpha_{1}^{n_{1}} & \ldots & \alpha_{k}^{n_{1}} \\
\ldots \ldots & \ldots & \ldots \\
\alpha_{1}^{n_{k}} & \ldots & \alpha_{k}^{n_{k}}
\end{array}\right|=0 .
$$

In analogy with our definition in Section 1 , we say that solutions $n_{1}, \ldots, n_{l}$ 
of (2.7) are in general position if in the matrix

$$
\left(\begin{array}{ccc}
\alpha_{1}^{n_{1}} & \ldots & \alpha_{k}^{n_{1}} \\
\ldots \ldots & \ldots & \ldots \\
\alpha_{1}^{n_{l}} & \ldots & \alpha_{k}^{n_{l}}
\end{array}\right)
$$

no subdeterminant of order $\leq k-1$ vanishes. We prove the following

TheOREM 2.1. Let $\mathbb{K}$ be an algebraically closed field of characteristic zero. Fix non-zero elements $\alpha_{1}, \ldots, \alpha_{k} \in \mathbb{K}$ such that $\alpha_{i} / \alpha_{j}$ is not a root of unity for $i \neq j$. Let $E$ be the set of equivalence classes of recurrence sequences $\left(u_{n}\right)_{n \in \mathbb{Z}}$ of minimal order $k$ and with characteristic roots $\alpha_{1}, \ldots, \alpha_{k}$. Then, apart from at most $\exp \left((6 k !)^{3 k !}\right)$ exceptional classes, any equivalence class in $E$ does not have more than $k-1$ zeros in general position.

By non-degeneracy, it is obvious that for $k=2$ any equivalence class in $E$ has at most one zero. Theorem 2.1 essentially says that for almost all equivalence classes of recurrence sequences the estimate (2.11) remains true in general.

Another consequence is the following. Let $\alpha_{1}, \ldots, \alpha_{k}$ be fixed elements in $\mathbb{K}$ satisfying (2.4) and (2.8). For any $k$-tuple $\boldsymbol{a}=\left(a_{1}, \ldots, a_{k}\right) \in \mathbb{K}^{k}$ with $a_{1} \ldots a_{k} \neq 0$, the vectors $\left(\alpha_{1}^{n}, \ldots, \alpha_{k}^{n}\right)$ solving the equation

$$
u_{n}=a_{1} \alpha_{1}^{n}+\ldots+a_{k} \alpha_{k}^{n}=0
$$

are contained in the linear subspace $H_{\boldsymbol{a}}$ of $\mathbb{K}^{k}$ defined by the equation

$$
a_{1} x_{1}+\ldots+a_{k} x_{k}=0 .
$$

It follows from (2.9) that the set of such vectors $\left(\alpha_{1}^{n}, \ldots, \alpha_{k}^{n}\right)$ is contained in the union of not more than $\exp \left(2(6 k)^{3 k}\right)$ one-dimensional linear subspaces of $H_{a}$. Our Theorem 2.1 implies that, apart from some exceptional cases, the above set is covered by a union of linear subspaces of codimension 1 in $H_{a}$ whose number does not exceed a bound simply exponential in $k$. We have

Corollary 2.2. Let $k \geq 3$. For all but at most $\exp \left((6 k !)^{3 k !}\right)$ equivalence classes of linear recurrence sequences

$$
u_{n}=a_{1} \alpha_{1}^{n}+\ldots+a_{k} \alpha_{k}^{n}
$$

of minimal order $k$ and with characteristic roots $\alpha_{1}, \ldots, \alpha_{k}$ satisfying (2.4) and (2.8), the vectors $\left(\alpha_{1}^{n}, \ldots, \alpha_{k}^{n}\right)$ such that $u_{n}=0$ are contained in the union of not more than

$$
\left(\begin{array}{c}
2 k-1 \\
k
\end{array}\right)-k(k-1)-2
$$

linear subspaces of codimension 1 in $H_{\boldsymbol{a}}$. 
We remark that the quantity (2.14) is less than

$$
2^{2 k-1} / \sqrt{k \pi} \text {. }
$$

It is clear that in the case $k=2$ our results are trivial. Notice that for $k=3$ the integer (2.14) is 2. For $k=3$, Evertse, Györy, Stewart and Tijdeman [3] have proved the following:

Let $\mathbb{K}$ be a number field. Let $S$ be a finite subset of the set of valuations of $\mathbb{K}$ containing the archimedean ones. Let $U_{S}$ be the group of $S$-units of $\mathbb{K}$. Two equations

$$
a_{1} x_{1}+a_{2} x_{2}+a_{3} x_{3}=0
$$

and

$$
a_{1}^{\prime} x_{1}+a_{2}^{\prime} x_{2}+a_{3}^{\prime} x_{3}=0
$$

are called $S$-equivalent if there exist $\lambda \in \mathbb{K}^{\times}$and $\varepsilon_{1}, \varepsilon_{2}, \varepsilon_{3} \in U_{S}$ such that $a_{i}^{\prime}=\lambda \varepsilon_{i} a_{i}$. In [3] it is shown that all but finitely many equivalence classes of $S$-unit equations (2.16) have not more than two projective solutions $\left(x_{1}\right.$ : $\left.x_{2}: x_{3}\right)$ with components in $U_{S}$.

In fact, if we have an equation (2.16) with three distinct projective solutions $\left(x_{1}: x_{2}: x_{3}\right),\left(y_{1}: y_{2}: y_{3}\right),\left(z_{1}: z_{2}: z_{3}\right)$ say, then

$$
\left|\begin{array}{lll}
x_{1} & x_{2} & x_{3} \\
y_{1} & y_{2} & y_{3} \\
z_{1} & z_{2} & z_{3}
\end{array}\right|=0
$$

and, as the solutions are distinct, in (2.17) no proper subdeterminant vanishes. So for cyclic groups $\Gamma$ our theorems generalize the result of [3] to arbitrary dimension $k$. We also mention in the context of Corollary 2.2 that for equivalence classes of $S$-unit equations (2.13) Evertse and Györy [2] have proved the following:

For all but finitely many $S$-equivalence classes of equations (2.13), the solutions in $S$-units of (2.13) are contained in the union of fewer than $2^{k !}$ $(k-2)$-dimensional linear subspaces of the subspace $H_{a}$ defined by $(2.13)$.

The bound $2^{k !}$ was improved by Evertse [1] to $(k-1) !^{2 k}$.

We can express our results more geometrically. Let $\Gamma$ be the cyclic subgroup of $\left(\mathbb{K}^{\times}\right)^{k}$ generated by $\left(\alpha_{1}, \ldots, \alpha_{k}\right)$. Let $H$ be a hyperplane in $\mathbb{K}^{k}$ defined by an equation

$$
a_{1} x_{1}+\ldots+a_{k} x_{k}=0
$$

with

$$
a_{1} \ldots a_{k} \neq 0 .
$$

We say that a hyperplane $H^{\prime}$ is equivalent $\bmod \Gamma$ to $H$ if there exists a point 
$\left(\alpha_{1}^{h}, \ldots, \alpha_{k}^{h}\right) \in \Gamma$ such that $H^{\prime}$ is defined by the equation

$$
\left(a_{1} \alpha_{1}^{h}\right) x_{1}+\ldots+\left(a_{k} \alpha_{k}^{h}\right) x_{k}=0 .
$$

We may phrase Theorem 2.1 in the following way:

COROLlary 2.3. There are at most $\exp \left((6 k !)^{3 k !}\right)$ equivalence classes of linear subspaces $H$ of codimension 1 in $\mathbb{K}^{k}$ defined by an equation (2.18) with (2.19) such that $H \cap \Gamma$ contains more than $k-1$ points $\left(\alpha_{1}^{n}, \ldots, \alpha_{k}^{n}\right)$ in general position.

3. Proof of Theorem 2.1 and of the corollaries. Theorem 2.1 is an easy consequence of Theorem 1.1. In fact, suppose that $\left(u_{n}\right)$ is a recurrence sequence that represents an exceptional equivalence class, i.e., suppose that the equation $u_{n}=0$ has more than $k-1$ solutions in general position. By equivalence, we may suppose that $u_{0}=0$, and we have $k-1$ further zeros $n_{2}, \ldots, n_{k}$ such that $0, n_{2}, \ldots, n_{k}$ are in general position. Therefore we get

$$
\left|\begin{array}{ccc}
1 & \ldots & 1 \\
\alpha_{1}^{n_{2}} & \ldots & \alpha_{k}^{n_{2}} \\
\ldots \ldots & \ldots & \ldots \\
\alpha_{1}^{n_{k}} & \ldots & \alpha_{k}^{n_{k}}
\end{array}\right|=0
$$

and here no proper subdeterminant vanishes. By Theorem 1.1, equation (3.1) has not more than $\exp \left((6 k !)^{3 k !}\right)$ solutions $\left(n_{2}, \ldots, n_{k}\right)$ in general position. However, given $n_{2}, \ldots, n_{k}$ as above, the coefficients $a_{1}, \ldots, a_{k}$ of $u_{n}$ in (2.5) are uniquely determined up to a common factor $a \neq 0$, and Theorem 2.1 follows.

As for Corollary 2.2, suppose $\left(u_{n}\right)$ represents a class that is not exceptional. Then the equation

$$
u_{n}=a_{1} \alpha_{1}^{n}+\ldots+a_{k} \alpha_{k}^{n}=0
$$

has $\leq k-1$ solutions in general position. Let $n_{1}, \ldots, n_{l}$ be a maximal set of solutions in general position, whence $l \leq k-1$. By the maximality of $l$, for any $n$ such that $u_{n}=0$ the matrix

$$
\left(\begin{array}{ccc}
\alpha_{1}^{n_{1}} & \ldots & \alpha_{k}^{n_{1}} \\
\ldots \ldots \ldots & \ldots . . \\
\alpha_{1}^{n_{l}} & \ldots & \alpha_{k}^{n_{l}} \\
\alpha_{1}^{n} & \ldots & \alpha_{k}^{n}
\end{array}\right)
$$

contains a square submatrix with vanishing determinant of an order $j$ such that

$$
2 \leq j \leq \min \{l+1, k-1\} .
$$

Since $n_{1}, \ldots, n_{l}$ are in general position, the intersection of the submatrix with the last row is non-empty. If the columns occurring in the submatrix 
have indices $h_{1}, \ldots, h_{j}$ and the rows preceding the last one have indices $i_{1}, \ldots, i_{j-1}$, we get

$$
\left|\begin{array}{ccc}
\alpha_{h_{1}}^{n_{i_{1}}} & \ldots & \alpha_{h_{j}}^{n_{i_{1}}} \\
\ldots \ldots \ldots \ldots & \ldots \ldots \ldots \\
\alpha_{h_{1}}^{n_{i_{j}-1}} & \ldots & \alpha_{h_{j}}^{n_{i_{j}}} \\
\alpha_{h_{1}}^{n} & \ldots & \alpha_{h_{j}}^{n}
\end{array}\right|=0 .
$$

For any choice of $h_{1}, \ldots, h_{j}$ and $i_{1}, \ldots, i_{j-1}$, equation (3.4) determines a linear subspace in $H_{\boldsymbol{a}}$ of dimension $k-2$ containing the vector $\left(\alpha_{1}^{n}, \ldots, \alpha_{k}^{n}\right)$. For a given $j$ we have $\left(\begin{array}{l}k \\ j\end{array}\right)$ choices for the columns and $\left(\begin{array}{c}l \\ j-1\end{array}\right)$ choices for the rows in (3.2). Summing over $j$ satisfying (3.3) we thus do not get more than

$$
\sum_{j=2}^{\min \{l+1, k-1\}}\left(\begin{array}{l}
k \\
j
\end{array}\right)\left(\begin{array}{c}
l \\
j-1
\end{array}\right)
$$

linear subspaces of dimension $k-2$.

For $l=k-2$ or $l=k-1$ we can do a little better. In these cases, the term in (3.5) corresponding to $j=k-1$ can be improved. Recall that the rows of the matrix (3.2) are solutions of

$$
\begin{gathered}
a_{1} x_{1}+\ldots+a_{k} x_{k}=0, \quad \text { where } \\
a_{1} \ldots a_{k} \neq 0 .
\end{gathered}
$$

Suppose first that $l=k-2$ and that in $(3.2)$ some $(k-1) \times(k-1)$ subdeterminant vanishes. Then it follows from (3.6) and (3.7) that every $(k-1) \times(k-1)$ subdeterminant vanishes. Consequently, the last row of (3.2) is a linear combination of the preceding rows. Therefore in the counting argument the term $\left(\begin{array}{c}k \\ k-1\end{array}\right)\left(\begin{array}{c}k-2 \\ k-2\end{array}\right)=k$ corresponding to $j=k-1$ in (3.5) can be replaced by 1 . Hence, for $l=k-2$, instead of (3.5) we get not more than

$$
1+\sum_{j=2}^{k-2}\left(\begin{array}{l}
k \\
j
\end{array}\right)\left(\begin{array}{l}
k-2 \\
j-1
\end{array}\right)
$$

linear subspaces of dimension $k-2$.

Now suppose $l=k-1$. Then the matrix (3.2) has $k-1$ submatrices of order $(k-1) \times k$ containing the row $\left(\alpha_{1}^{n}, \ldots, \alpha_{k}^{n}\right)$. For each such submatrix we are in the situation treated for $l=k-2$. Therefore in (3.5) the term $\left(\begin{array}{c}k \\ k-1\end{array}\right)\left(\begin{array}{c}k-1 \\ k-2\end{array}\right)=k(k-1)$ corresponding to $j=k-1$ can be replaced by $k-1$. Consequently, for $l=k-1$ we get not more than

$$
k-1+\sum_{j=2}^{k-2}\left(\begin{array}{l}
k \\
j
\end{array}\right)\left(\begin{array}{l}
k-1 \\
j-1
\end{array}\right)
$$

linear subspaces of dimension $k-2$. 
We now compare the bounds (3.5), (3.8) and (3.9). For $l \leq k-3$ we get

$$
\sum_{j=2}^{\min \{l+1, k-1\}}\left(\begin{array}{l}
k \\
j
\end{array}\right)\left(\begin{array}{c}
l \\
j-1
\end{array}\right) \leq \sum_{j=2}^{k-2}\left(\begin{array}{l}
k \\
j
\end{array}\right)\left(\begin{array}{c}
k-3 \\
j-1
\end{array}\right) .
$$

Combining this with (3.8) and (3.9), we see that in any case $\left(\alpha_{1}^{n}, \ldots, \alpha_{k}^{n}\right)$ is contained in the union of not more than

$$
k-1+\sum_{j=2}^{k-2}\left(\begin{array}{l}
k \\
j
\end{array}\right)\left(\begin{array}{l}
k-1 \\
j-1
\end{array}\right)=k-1+\sum_{j=2}^{k-2}\left(\begin{array}{l}
k \\
j
\end{array}\right)\left(\begin{array}{l}
k-1 \\
k-j
\end{array}\right)
$$

linear subspaces of dimension $k-2$.

Note that if $A$ and $B$ are sets containing $a$ and $b$ elements respectively with $A \cap B=\emptyset$, choosing $j$ elements in $A$ and $k-j$ elements in $B$ gives $k$ elements in $A \cup B$. Hence

$$
\sum_{j=\max \{0, k-b\}}^{\min \{k, a\}}\left(\begin{array}{l}
a \\
j
\end{array}\right)\left(\begin{array}{c}
b \\
k-j
\end{array}\right)=\left(\begin{array}{c}
a+b \\
k
\end{array}\right) .
$$

In particular we have

$$
\sum_{j=1}^{k}\left(\begin{array}{l}
k \\
j
\end{array}\right)\left(\begin{array}{l}
k-1 \\
k-j
\end{array}\right)=\left(\begin{array}{c}
2 k-1 \\
k
\end{array}\right),
$$

whence

$$
k-1+\sum_{j=2}^{k-2}\left(\begin{array}{l}
k \\
j
\end{array}\right)\left(\begin{array}{l}
k-1 \\
k-j
\end{array}\right)=\left(\begin{array}{c}
2 k-1 \\
k
\end{array}\right)-k(k-1)-2 .
$$

This proves Corollary 2.2.

Also

$$
\frac{\sqrt{k}}{2^{2 k-1}}\left(\begin{array}{c}
2 k-1 \\
k
\end{array}\right)
$$

is an increasing sequence with limit $\pi^{-1 / 2}$ by Stirling's formula. Therefore

$$
\left(\begin{array}{c}
2 k-1 \\
k
\end{array}\right)<\frac{2^{2 k-1}}{\sqrt{k \pi}}
$$

and the bound (2.15) follows.

Corollary 2.3 is a direct consequence of Theorem 2.1.

4. Relation groups. Here and in the next sections $\mathbb{K}$ denotes a field of characteristic zero.

We begin by quoting Theorem 1.1 of Evertse, Schlickewei and Schmidt [4], which for convenience we state as a lemma. 
Lemma 4.1. Let $n$ be a positive integer, and let $\Gamma$ be a finitely generated subgroup of rank $r$ of the multiplicative group $\left(\mathbb{K}^{\times}\right)^{n}$. Let $a_{1}, \ldots, a_{n}$ be nonzero elements in $\mathbb{K}$. Then the equation

$$
a_{1} x_{1}+\ldots+a_{n} x_{n}+1=0
$$

does not have more than

$$
\exp \left((6 n)^{3 n}(r+1)\right)
$$

non-degenerate solutions $\boldsymbol{x}=\left(x_{1}, \ldots, x_{n}\right) \in \Gamma$.

(A solution $\left(x_{1}, \ldots, x_{n}\right)$ of $(4.1)$ is non-degenerate if $\sum_{i \in I} a_{i} x_{i} \neq 0$ for any non-empty subset $I$ of $\{1, \ldots, n\}$.)

Now suppose that we are given non-zero elements in $\mathbb{K}$ :

$$
\beta_{11}, \ldots, \beta_{1 r} ; \ldots ; \beta_{n 1}, \ldots, \beta_{n r} ; \beta_{n+1,1}, \ldots, \beta_{n+1, r}
$$

and consider the equation

$$
b_{1} \boldsymbol{\beta}_{1}^{m}+\ldots+b_{n+1} \boldsymbol{\beta}_{n+1}^{m}=0
$$

where $\boldsymbol{\beta}_{l}^{m}=\beta_{l 1}^{m_{1}} \ldots \beta_{l r}^{m_{r}}(1 \leq l \leq n+1), \boldsymbol{m}=\left(m_{1}, \ldots, m_{r}\right) \in \mathbb{Z}^{r}$, and where $b_{1}, \ldots, b_{n+1}$ are given non-zero elements in $\mathbb{K}$. We apply Lemma 4.1 to equation (4.2).

Let $\mathcal{P}$ be a partition of the set $\Lambda=\{1, \ldots, n+1\}$. The sets $\lambda \subset \Lambda$ occurring in $\mathcal{P}$ will be considered elements of $\mathcal{P}: \lambda \in \mathcal{P}$. Given $\mathcal{P}$, the system of equations

$$
\sum_{l \in \lambda} b_{l} \boldsymbol{\beta}_{l}^{m}=0 \quad(\lambda \in \mathcal{P})
$$

is a refinement of (4.2). If $\mathcal{Q}$ is a refinement of $\mathcal{P}$, then $(4.2 \mathcal{Q})$ implies $(4.2 \mathcal{P})$. Write $\mathfrak{S}(\mathcal{P})$ for the set of solutions $\boldsymbol{m} \in \mathbb{Z}^{r}$ of $(4.2 \mathcal{P})$ which are not solutions of $(4.2 \mathcal{Q})$ for any proper refinement $\mathcal{Q}$ of $\mathcal{P}$.

For a given $\lambda \in \mathcal{P}$, the equation

$$
\sum_{l \in \lambda} b_{l} \boldsymbol{\beta}_{l}^{m}=0
$$

has solution vectors $\left(\boldsymbol{\beta}_{l}^{m}\right)_{l \in \lambda}=\left(\beta_{l 1}^{m_{1}} \ldots \beta_{l r}^{m_{r}}\right)_{l \in \lambda}$ in a subgroup $\Gamma_{\lambda}$ of $\left(\mathbb{K}^{\times}\right)^{|\lambda|}$ of rank $\leq r$. By homogeneity and in view of Lemma 4.1, the number $C(\lambda)$ of non-proportional non-degenerate solutions $\left(\boldsymbol{\beta}_{l}^{m}\right)_{l \in \lambda}$ of equation (4.3) satisfies

$$
C(\lambda) \leq \exp \left((6(|\lambda|-1))^{3(|\lambda|-1)}(r+1)\right) .
$$

For simplicity of notation, suppose that $\lambda=\{1, \ldots, h+1\}$. We associate with (4.3) the inhomogeneous equation

$$
c_{1} \gamma_{1}^{m}+\ldots+c_{h} \gamma_{h}^{m}+1=0,
$$


where $c_{l}=b_{l} / b_{h+1}$ and $\boldsymbol{\gamma}_{l}^{\boldsymbol{m}}=\boldsymbol{\beta}_{l}^{\boldsymbol{m}} / \boldsymbol{\beta}_{h+1}^{m}=\left(\beta_{l 1} / \beta_{h+1,1}\right)^{m_{1}} \ldots\left(\beta_{l r} / \beta_{h+1, r}\right)^{m_{r}}$ for $1 \leq l \leq h$.

Let $\left(\gamma_{1}^{m_{j}}, \ldots, \gamma_{h}^{m_{j}}\right)(1 \leq j \leq C(\lambda))$ be an enumeration of the nondegenerate solutions of (4.5). Then any non-degenerate solution $\left(\boldsymbol{\beta}_{l}^{\boldsymbol{m}}\right)_{l \in \lambda}$ of (4.3) will be of the form

$$
\left(\boldsymbol{\beta}_{l}^{\boldsymbol{m}}\right)_{l \in \lambda}=\psi_{\lambda}(\boldsymbol{m})\left(\gamma_{1}^{\boldsymbol{m}_{j}}, \ldots, \gamma_{h}^{\boldsymbol{m}_{j}}, 1\right)
$$

for a suitable $j$ with $1 \leq j \leq C(\lambda)$ and a factor of proportionality $\psi_{\lambda}(\boldsymbol{m})=$ $\boldsymbol{\beta}_{h+1}^{\boldsymbol{m}}$. If two exponents $\boldsymbol{m}$ and $\boldsymbol{m}^{\prime}$ in (4.3) give rise to the same tuple $\left(\gamma_{1}^{m_{j}}, \ldots, \gamma_{h}^{m_{j}}, 1\right)$ in $(4.6)$, we may conclude that

$$
\left(\frac{\boldsymbol{\beta}_{1}^{m}}{\psi_{\lambda}(\boldsymbol{m})}, \ldots, \frac{\boldsymbol{\beta}_{h+1}^{m}}{\psi_{\lambda}(\boldsymbol{m})}\right)=\left(\frac{\boldsymbol{\beta}_{1}^{\boldsymbol{m}^{\prime}}}{\psi_{\lambda}\left(\boldsymbol{m}^{\prime}\right)}, \ldots, \frac{\boldsymbol{\beta}_{h+1}^{m^{\prime}}}{\psi_{\lambda}\left(\boldsymbol{m}^{\prime}\right)}\right)
$$

and therefore we obtain

$$
\frac{\psi_{\lambda}(\boldsymbol{m})}{\psi_{\lambda}\left(\boldsymbol{m}^{\prime}\right)}=\boldsymbol{\beta}_{1}^{m-\boldsymbol{m}^{\prime}}=\ldots=\boldsymbol{\beta}_{h+1}^{m-\boldsymbol{m}^{\prime}}
$$

However, for a partition $\mathcal{P}$ of $\Lambda$ and for $\boldsymbol{m}, \boldsymbol{m}^{\prime} \in \mathfrak{S}(\mathcal{P})$, the above considerations have to be done simultaneously for all sets $\lambda \in \mathcal{P}$. Each set $\lambda$ will produce relations (4.7), and so we have to deal with relations (4.7) simultaneously for each $\lambda \in \mathcal{P}$.

Given a partition $\mathcal{P}$ of $\Lambda$ and $l, l^{\prime} \in \Lambda$, write $l \stackrel{\mathcal{P}}{\sim} l^{\prime}$ if there exists a set $\lambda \in \mathcal{P}$ such that $l, l^{\prime} \in \lambda$. Define the subgroup $G(\mathcal{P})$ of the additive group $\mathbb{Z}^{r}$ as the set of tuples $\boldsymbol{z}=\left(z_{1}, \ldots, z_{r}\right) \in \mathbb{Z}^{r}$ satisfying

$$
\boldsymbol{\beta}_{l}^{z}=\boldsymbol{\beta}_{l^{\prime}}^{z} \quad \text { for any } l, l^{\prime} \in \Lambda \text { such that } l \stackrel{\mathcal{P}}{\sim} l^{\prime} .
$$

In view of (4.7), we may infer that if $G(\mathcal{P})=\{\mathbf{0}\}$, then $\mathfrak{S}(\mathcal{P})$ does not contain more than $\prod_{\lambda \in \mathcal{P}} C(\lambda)$ elements. By (4.4) this implies

Lemma 4.2. Let $\mathcal{P}$ be a partition of $\Lambda=\{1, \ldots, n+1\}$ such that $G(\mathcal{P})=\{\mathbf{0}\}$. Then equation (4.2) does not have more than $\exp \left((6 n)^{3 n}(r+1)\right)$ solutions $\boldsymbol{m} \in \mathfrak{S}(\mathcal{P})$.

5. Relation groups for equation (1.3). Equation (1.3) is

$$
\left|\begin{array}{ccc}
1 & \ldots & 1 \\
\alpha_{1}^{y_{2}} & \ldots & \alpha_{k}^{y_{2}} \\
\ldots \ldots \ldots & \ldots & \ldots \\
\alpha_{1}^{y_{k}} & \ldots & \alpha_{k}^{y_{k}}
\end{array}\right|=0
$$

with $\alpha_{1}, \ldots, \alpha_{k} \in \mathbb{K}^{\times}$such that $\alpha_{i} / \alpha_{j}$ is not a root of unity for $i \neq j$. We write (5.1) as

$$
\sum_{\sigma \in \mathfrak{S}_{k}}(\operatorname{sign} \sigma) \alpha_{\sigma(1)}^{y_{1}} \ldots \alpha_{\sigma(k)}^{y_{k}}=0
$$


where $\mathfrak{S}_{k}$ denotes the symmetric group of the permutations of $\{1, \ldots, k\}$ and where $y_{1}=0$.

We apply the material from Section 4 to equation (5.2). Initially, this is an equation in $\left(y_{2}, \ldots, y_{k}\right) \in \mathbb{Z}^{k-1}$; however, in the following discussion it is convenient to view it as an equation in $\left(y_{1}, y_{2}, \ldots, y_{k}\right) \in \mathbb{Z}^{k}$ with the convention that $y_{1}=0$, as indicated above.

Given a partition $\mathcal{P}$ of $\mathfrak{S}_{k}$, let $\mathfrak{S}(\mathcal{P})$ denote, in accordance with $(4.2 \mathcal{P})$, the set of solutions $\left(y_{1}, y_{2}, \ldots, y_{k}\right)=\left(0, y_{2}, \ldots, y_{k}\right) \in \mathbb{Z}^{k}$ of the system of equations

$$
\sum_{\sigma \in \lambda}(\operatorname{sign} \sigma) \alpha_{\sigma(1)}^{y_{1}} \ldots \alpha_{\sigma(k)}^{y_{k}}=0 \quad(\lambda \in \mathcal{P})
$$

which are not solutions of any system $(5.2 \mathcal{Q})$ corresponding to a proper refinement $\mathcal{Q}$ of $\mathcal{P}$.

The analogue of (4.8) in the present context is

$$
\alpha_{\sigma(1)}^{z_{1}} \ldots \alpha_{\sigma(k)}^{z_{k}}=\alpha_{\tau(1)}^{z_{1}} \ldots \alpha_{\tau(k)}^{z_{k}} \quad \text { for any } \sigma, \tau \in \mathfrak{S}_{k} \text { with } \sigma \stackrel{\mathcal{P}}{\sim} \tau .
$$

Hence we define $G(\mathcal{P})$ as the additive group of the $k$-tuples $\left(z_{1}, z_{2}, \ldots, z_{k}\right)=$ $\left(0, z_{2}, \ldots, z_{k}\right) \in \mathbb{Z}^{k}$ satisfying (5.3).

In view of Lemma 4.2 , we are now interested in the shape of partitions $\mathcal{P}$ such that $G(\mathcal{P}) \neq\{\mathbf{0}\}$. Let $\mathcal{P}$ be such a partition. Thus there exists a $k$-tuple $\left(w_{1}, w_{2}, \ldots, w_{k}\right)=\left(0, w_{2}, \ldots, w_{k}\right) \in G(\mathcal{P})$ with $w_{2}, \ldots, w_{k}$ not all zero, so that (5.3) holds with $z_{i}=w_{i}$. This condition entails a structure in suitable sets $\lambda \in \mathcal{P}$. Precisely, we claim that, up to a suitable renumbering of $\alpha_{1}, \ldots, \alpha_{k}$, there are sets $\lambda_{1}, \ldots, \lambda_{s} \in \mathcal{P}(s \geq 1)$ such that their union

$$
\lambda_{*}=\lambda_{1} \cup \ldots \cup \lambda_{s}
$$

coincides with the set of permutations $\sigma \in \mathfrak{S}_{k}$ for which

$$
w_{\sigma^{-1}(1)} \leq w_{\sigma^{-1}(2)} \leq \ldots \leq w_{\sigma^{-1}(k)} .
$$

Let $\delta_{1}, \ldots, \delta_{k} \in \mathbb{K}^{\times}$be multiplicatively independent elements such that

$$
\alpha_{i}=\zeta_{i} \delta_{1}^{a_{i 1}} \ldots \delta_{k}^{a_{i k}} \quad(i=1, \ldots, k),
$$

where $a_{i 1}, \ldots, a_{i k}$ are suitable integers and $\zeta_{i}$ is a root of unity for $i=$ $1, \ldots, k$. Write

$$
\boldsymbol{a}_{i}=\left(\begin{array}{c}
a_{i 1} \\
\vdots \\
a_{i k}
\end{array}\right) .
$$

Substituting (5.5) into

$$
\alpha_{\sigma(1)}^{w_{1}} \ldots \alpha_{\sigma(k)}^{w_{k}}=\alpha_{\tau(1)}^{w_{1}} \ldots \alpha_{\tau(k)}^{w_{k}}
$$


and comparing exponents, we obtain

$$
w_{1} \boldsymbol{a}_{\sigma(1)}+\ldots+w_{k} \boldsymbol{a}_{\sigma(k)}=w_{1} \boldsymbol{a}_{\tau(1)}+\ldots+w_{k} \boldsymbol{a}_{\tau(k)} \quad \text { for } \sigma \stackrel{\mathcal{P}}{\sim} \tau .
$$

Note that, since $\alpha_{i} / \alpha_{j}$ is not a root of unity for $i \neq j$, the vectors $\boldsymbol{a}_{1}, \ldots, \boldsymbol{a}_{k}$ are distinct. Pick a vector $\boldsymbol{v} \in \mathbb{R}^{k}$ such that the inner products $c_{i}=\boldsymbol{a}_{i} \cdot \boldsymbol{v}$ $(i=1, \ldots, k)$ are distinct. We may assume without loss of generality that

$$
c_{1}<\ldots<c_{k} .
$$

Otherwise it suffices to renumber $\alpha_{1}, \ldots, \alpha_{k}$. The inner product by $\boldsymbol{v}$ in (5.6) yields

$$
w_{1} c_{\sigma(1)}+\ldots+w_{k} c_{\sigma(k)}=w_{1} c_{\tau(1)}+\ldots+w_{k} c_{\tau(k)},
$$

i.e.,

$$
w_{\sigma^{-1}(1)} c_{1}+\ldots+w_{\sigma^{-1}(k)} c_{k}=w_{\tau^{-1}(1)} c_{1}+\ldots+w_{\tau^{-1}(k)} c_{k} \quad \text { for } \sigma \stackrel{\mathcal{P}}{\sim} \tau .
$$

Let $s$ be the maximal number of permutations $\sigma$ inequivalent under $\mathcal{P}$ and satisfying (5.4). Take $s$ permutations $\sigma_{1}, \ldots, \sigma_{s}$, say, with this property, and let $\lambda_{1}, \ldots, \lambda_{s} \in \mathcal{P}$ be such that $\sigma_{j} \in \lambda_{j}(j=1, \ldots, s)$. Then $\lambda_{1}, \ldots, \lambda_{s}$ are distinct. By (5.7), the quantity $w_{\sigma^{-1}(1)} c_{1}+\ldots+w_{\sigma^{-1}(k)} c_{k}$ is maximal for $\sigma \in \mathfrak{S}_{k}$ if and only if (5.4) holds. In particular we know that, for any $j=1, \ldots, s$,

$$
w_{\sigma_{j}^{-1}(1)} c_{1}+\ldots+w_{\sigma_{j}^{-1}(k)} c_{k}
$$

takes the same maximal value. By (5.8) and by the maximality of $s$,

$$
w_{\tau^{-1}(1)} c_{1}+\ldots+w_{\tau^{-1}(k)} c_{k}
$$

is maximal if and only if

$$
\tau \in \lambda_{*}=\lambda_{1} \cup \ldots \cup \lambda_{s} .
$$

We may infer that $\lambda_{*}$ is the set of permutations $\sigma \in \mathfrak{S}_{k}$ satisfying (5.4), as claimed.

Now consider the partition $\left\{\eta_{1}, \ldots, \eta_{m}\right\}$ of $\{1, \ldots, k\}$ defined by

$$
\begin{array}{ll}
w_{i}=w_{j} & \text { for all } i, j \in \eta_{\mu}, \mu=1, \ldots, m, \\
w_{i}<w_{j} & \text { for all } i \in \eta_{\mu}, j \in \eta_{\mu^{\prime}}, \mu<\mu^{\prime} .
\end{array}
$$

Note that $m \geq 2$, for otherwise $0=w_{1}=\ldots=w_{k}$, contradicting the assumption that $w_{2}, \ldots, w_{k}$ are not all zero. Moreover, let $\left\{\vartheta_{1}, \ldots, \vartheta_{m}\right\}$ be the partition of $\{1, \ldots, k\}$ such that

$$
\left|\vartheta_{\mu}\right|=\left|\eta_{\mu}\right| \quad(\mu=1, \ldots, m)
$$

and

$$
i<j \quad \text { for all } i \in \vartheta_{\mu}, j \in \vartheta_{\mu^{\prime}}, \mu<\mu^{\prime} \text {. }
$$


In other words we have, for any $\mu=1, \ldots, m$,

$$
\vartheta_{\mu}=\left\{\sum_{\varrho=1}^{\mu-1}\left|\eta_{\varrho}\right|+1, \sum_{\varrho=1}^{\mu-1}\left|\eta_{\varrho}\right|+2, \ldots, \sum_{\varrho=1}^{\mu-1}\left|\eta_{\varrho}\right|+\left|\eta_{\mu}\right|\right\} .
$$

Clearly the permutations $\sigma \in \mathfrak{S}_{k}$ satisfying (5.4) are those for which

$$
\sigma: \eta_{\mu} \rightarrow \vartheta_{\mu} \quad(\mu=1, \ldots, m) .
$$

Hence $\sigma \in \lambda_{*}$ if and only if (5.9) holds.

We now assume $\mathfrak{S}(\mathcal{P}) \neq \emptyset$. Let $\left(0, y_{2}, \ldots, y_{k}\right) \in \mathfrak{S}(\mathcal{P})$. By $(5.2 \mathcal{P})$ we have

$$
\sum_{\sigma \in \lambda_{j}}(\operatorname{sign} \sigma) \alpha_{\sigma(1)}^{y_{1}} \ldots \alpha_{\sigma(k)}^{y_{k}}=0 \quad(j=1, \ldots, s),
$$

whence, summing over $j=1, \ldots, s$,

$$
\sum_{\sigma \in \lambda_{*}}(\operatorname{sign} \sigma) \alpha_{\sigma(1)}^{y_{1}} \ldots \alpha_{\sigma(k)}^{y_{k}}=0 .
$$

Since $\lambda_{*}$ is the set of $\sigma \in \mathfrak{S}_{k}$ satisfying (5.9), we obtain

$$
\sum_{\sigma \in \lambda_{*}}(\operatorname{sign} \sigma) \alpha_{\sigma(1)}^{y_{1}} \ldots \alpha_{\sigma(k)}^{y_{k}}= \pm \prod_{\mu=1}^{m} \operatorname{det}\left(\alpha_{h}^{y_{i}}\right)_{h \in \vartheta_{\mu}, i \in \eta_{\mu}} .
$$

By (5.10) this product vanishes. Hence for at least one $\mu(1 \leq \mu \leq m)$ we have

$$
\operatorname{det}\left(\alpha_{h}^{y_{i}}\right)_{h \in \vartheta_{\mu}, i \in \eta_{\mu}}=0 .
$$

This is a proper subdeterminant of (5.1), since $m \geq 2$ and therefore $\left|\vartheta_{\mu}\right|=$ $\left|\eta_{\mu}\right|<k$.

We have proved

Lemma 5.1. Let $\mathcal{P}$ be a partition of $\mathfrak{S}_{k}$ with $G(\mathcal{P}) \neq\{\mathbf{0}\}$. Then for each solution $\left(y_{2}, \ldots, y_{k}\right) \in \mathbb{Z}^{k-1}$ of (5.1) such that $\left(0, y_{2}, \ldots, y_{k}\right) \in \mathfrak{S}(\mathcal{P})$, a proper subdeterminant of the left-hand side of (5.1) vanishes.

6. Proof of Theorem 1.1. We use Lemmas 4.2 and 5.1. Equation (1.3), in view of (5.2) where $y_{1}=0$, is an instance of (4.2) with $r=k-1$ and $n=k !-1$.

By Lemma 4.2 , any partition $\mathcal{P}$ of $\mathfrak{S}_{k}$ with $G(\mathcal{P})=\{\mathbf{0}\}$ satisfies

$$
|\mathfrak{S}(\mathcal{P})| \leq \exp \left((6(k !-1))^{3(k !-1)} k\right) .
$$

On the other hand, by Lemma 5.1, partitions $\mathcal{P}$ with $G(\mathcal{P}) \neq\{\mathbf{0}\}$ are such that for any solution in $\mathfrak{S}(\mathcal{P})$ a proper subdeterminant vanishes. The number of partitions of $\mathfrak{S}_{k}$ is $\leq k !^{k !}$. Combining this with (6.1) we see that in fact (1.3) does not have more than $\exp \left((6 k !)^{3 k !}\right)$ solutions in general position. 


\section{References}

[1] J.-H. Evertse, Decomposable form equations with a small linear scattering, J. Reine Angew. Math. 432 (1992), 177-217.

[2] J.-H. Evertse and K. Györy, On the numbers of solutions of weighted unit equations, Compositio Math. 66 (1988), 329-354.

[3] J.-H. Evertse, K. Győry, C. L. Stewart and R. Tijdeman, On S-unit equations in two unknowns, Invent. Math. 92 (1988), 461-477.

[4] J.-H. Evertse, H. P. Schlickewei and W. M. Schmidt, Linear equations in variables which lie in a multiplicative group, submitted to Ann. of Math.

Fachbereich Mathematik

Universität Marburg

Dipartimento di Matematica

Hans-Meerwein-Straße

Università di Pisa

Lahnberge

Via Buonarroti 2

56127 Pisa, Italy

35032 Marburg/Lahn, Germany

E-mail: viola@dm.unipi.it

E-mail: hps@mathematik.uni-marburg.de

Received on 2.6.1998

and in revised form on 16.11.1998

$(3400)$ 